\title{
Group Space Scan of Flavor Symmetries for Nearly Tribimaximal Lepton Mixing
}

\author{
Florian Plentingert, Gerhart Seid团, and Walter Winter约 \\ Institut für Theoretische Physik und Astrophysik \\ Universität Würzburg, D-97074 Würzburg, Germany
}

\begin{abstract}
We present a systematic group space scan of discrete Abelian flavor symmetries for lepton mass models that produce nearly tribimaximal lepton mixing. In our models, small neutrino masses are generated by the type-I seesaw mechanism. The lepton mass matrices emerge from higher-dimension operators via the Froggatt-Nielsen mechanism and are predicted as powers of a single expansion parameter $\epsilon$ that is of the order of the Cabibbo angle $\theta_{\mathrm{C}} \simeq 0.2$. We focus on solutions that can give close to tribimaximal lepton mixing with a very small reactor angle $\theta_{13} \approx 0$ and find several thousand explicit such models that provide an excellent fit to current neutrino data. The models are rather general in the sense that large leptonic mixings can come from the charged leptons and/or neutrinos. Moreover, in the neutrino sector, both left- and right-handed neutrinos can mix maximally. We also find a new relation $\theta_{13} \lesssim \mathcal{O}\left(\epsilon^{3}\right)$ for the reactor angle and a new sum rule $\theta_{23} \approx \frac{\pi}{4}+\epsilon / \sqrt{2}$ for the atmospheric angle, allowing the models to be tested in future neutrino oscillation experiments.
\end{abstract}

\footnotetext{
${ }^{\dagger}$ E-mail: fplentinger@physik.uni-wuerzburg.de

${ }_{\ddagger}^{\ddagger}$ E-mail: seidl@physik.uni-wuerzburg. de

${ }^{\S}$ E-mail: winter@physik.uni-wuerzburg.de
} 


\section{Introduction}

During the past decade, solar [1], atmospheric [2, reactor [3], and accelerator [4] neutrino oscillation experiments, have very well established that neutrinos are massive. Since neutrinos are massless in the standard model (SM), massive neutrinos signal physics beyond the $\mathrm{SM}$. In fact, the smallness of neutrino masses $\sim 10^{-2} \ldots 10^{-1} \mathrm{eV}$ can be naturally connected with grand unified theories (GUTs) [5, 6] via the seesaw mechanism [7, 8], in which the absolute neutrino mass scale becomes suppressed by an energy scale close to the GUT scale $M_{\mathrm{GUT}} \approx 2 \times 10^{16} \mathrm{GeV}[9]$.

Current neutrino oscillation data (for a recent global fit see Ref. [10]) tells us that the Pontecorvo-Maki-Nakagawa-Sakata (PMNS) lepton mixing matrix $U_{\text {PMNS }}$ [1] can be approximated by the Harrison-Perkins-Scott (HPS) tribimaximal mixing matrix $U_{\mathrm{HPS}}$ [12] as

$$
U_{\mathrm{PMNS}} \approx U_{\mathrm{HPS}}=\left(\begin{array}{ccc}
\sqrt{\frac{2}{3}} & \frac{1}{\sqrt{3}} & 0 \\
-\frac{1}{\sqrt{6}} & \frac{1}{\sqrt{3}} & -\frac{1}{\sqrt{2}} \\
-\frac{1}{\sqrt{6}} & \frac{1}{\sqrt{3}} & \frac{1}{\sqrt{2}}
\end{array}\right) .
$$

In $U_{\mathrm{HPS}}$, the solar angle $\theta_{12}$ and the atmospheric angle $\theta_{23}$ are given by $\theta_{12}=\arctan (1 / \sqrt{2})$ and $\theta_{23}=\pi / 4$, whereas the reactor angle $\theta_{13}$ vanishes, i.e., $\theta_{13}=0$. The actually observed leptonic mixing angles in $U_{\text {PMNS }}$ may then be expressed in terms of deviations from tribimaximal mixing [13, 14] as "nearly" or "near" tribimaximal lepton mixing [15].

Many models have been proposed in the literature to reproduce tribimaximal leptonic mixing using non-Abelian flavor symmetries. These models, however, have generally difficulties (for a discussion see Ref. [16]) to predict the observed fermion mass hierarchies and the Cabibbo-Kobayashi-Maskawa (CKM) quark mixing matrix $V_{\mathrm{CKM}}$ [17] in the context of the canonical type-I seesaw mechanism (see Ref. [18] for models including quarks and Ref. [19] for unified models). An interesting connection between the quark and the lepton sector is implied by the idea of quark-lepton complementarity (QLC) [20], which is the phenomenological observation that the measured solar mixing angle very accurately satisfies the relation $\theta_{12}+\theta_{\mathrm{C}} \approx \pi / 4$, where $\theta_{\mathrm{C}} \simeq 0.2$ is the Cabibbo angle. QLC has been studied from many different points of view: as a correction to bimaximal mixing [21, together with sum rules [22], with stress on phenomenological aspects [23], in conjunction with parameterizations of $U_{\text {PMNS }}$ in terms of $\theta_{\mathrm{C}}$ [24], with respect to statistical arguments [25], by including renormalization group effects [26], and in model building realizations [27].

In Refs. [28, 29], we have suggested a generalization of QLC to "extended QLC" (EQLC), where the mixing angles of the left- and right-handed leptons can assume any of the values $\frac{\pi}{4}, \epsilon, \epsilon^{2}, 0$. Here, $\epsilon$ is of the order of the Cabibbo angle $\epsilon \simeq 0.2$. By expressing also the lepton mass ratios as powers of $\epsilon$, we have derived in Ref. [29] for the CP-conserving case (a discussion of nonzero phases can be found in Ref. [30]) in total 1981 qualitatively distinct mass matrix textures for the charged leptons and neutrinos that lead to nearly tribimaximal neutrino mixing with a small reactor angle $\theta_{13} \approx 0$. For these textures, the neutrino masses become small due to the canonical type-I seesaw mechanism (for a related approach see Ref. [31]). The matrix elements of these textures are in the flavor basis all expressed by powers of $\epsilon$, which serves as a single small expansion parameter of the matrices. This 
suggests a model building interpretation of the textures in terms of flavor symmetries, e.g. via the Froggatt-Nielsen mechanism [32].

In this paper, we describe the systematic construction of several thousand explicit lepton mass models, in which nearly tribimaximal lepton mixing and the mass hierarchies of charged leptons and neutrinos emerge from products of discrete Abelian flavor symmetries. Our motivation is that models with Abelian flavor symmetries generally have the merit that they need only a very simple scalar sector to achieve the necessary flavor symmetry breaking. We perform a group space scan of products of discrete $Z_{n}$ flavor symmetries using the results of EQLC from Ref. [29] and restrict to the case of real lepton mass matrices, i.e., we consider the CP-conserving case. In order to generate small neutrino masses, we assume only the canonical type-I seesaw mechanism. The hierarchical pattern of the lepton mass matrices results from higher-dimension operators that are produced by the Froggatt-Nielsen mechanism. Moreover, we are interested only in flavor symmetries that yield nearly tribimaximal lepton mixing with a very small reactor angle $\theta_{13} \approx 0$.

The paper is organized as follows: In Sec. 2, we introduce the notation for the lepton masses and mixings. Next, in Sec. 3, we specify our discrete flavor symmetries and describe the generation of lepton mass terms via the Froggatt-Nielsen mechanism. Then, we outline in Sec. 4 our method for scanning the group space of flavor symmetries. Our general results, a list of explicit example models, a new relation for the reactor angle, and our sum rules for the PMNS angles including a new sum rule for the atmospheric angle are shown in Sec. 5. Finally, we present in Sec. 6 our summary and conclusions.

\section{Lepton Masses and Mixings}

We assume the SM with gauge group $G_{\mathrm{SM}}=S U(3)_{c} \times S U(2)_{L} \times U(1)_{Y}$ plus three righthanded neutrinos that generate small neutrino masses via the type-I seesaw mechanism [7]. The lepton Yukawa couplings and mass terms are

$$
\mathcal{L}_{\mathrm{Y}}=-\left(Y_{\ell}\right)_{i j} H^{*} \ell_{i} e_{j}^{c}-\left(Y_{D}\right)_{i j} \mathrm{i} \sigma^{2} H \ell_{i} \nu_{j}^{c}-\frac{1}{2}\left(M_{R}\right)_{i j} \nu_{i}^{c} \nu_{j}^{c}+\text { h.c. }
$$

where $\ell_{i}=\left(\nu_{i}, e_{i}\right)^{T}, e_{i}^{c}$, and $\nu_{i}^{c}$, are the left-handed leptons, the right-handed charged lepton doublets, and the right-handed SM singlet neutrinos, and $i=1,2,3$ is the generation index. Here, $H$ is the SM Higgs doublet, $Y_{\ell}$ and $Y_{D}$ are the Dirac Yukawa coupling matrices of the charged leptons and neutrinos, and $M_{R}$ is the Majorana mass matrix of the right-handed neutrinos with entries of the order of the $B-L$ breaking scale $M_{B-L} \sim 10^{14} \mathrm{GeV}$. After electroweak symmetry breaking, $H$ develops a vacuum expectation value $\langle H\rangle \sim 10^{2} \mathrm{GeV}$, and the mass terms of the leptons become

$$
\mathcal{L}_{\text {mass }}=-\left(M_{\ell}\right)_{i j} e_{i} e_{j}^{c}-\left(M_{D}\right)_{i j} \nu_{i} \nu_{j}^{c}-\frac{1}{2}\left(M_{R}\right)_{i j} \nu_{i}^{c} \nu_{j}^{c}+\text { h.c. },
$$

where $M_{\ell}=\langle H\rangle Y_{\ell}$ is the charged lepton and $M_{D}=\langle H\rangle Y_{D} \sim 10^{2} \mathrm{GeV}$ the Dirac neutrino mass matrix. After integrating out the right-handed neutrinos, the seesaw mechanism leads to the effective Majorana neutrino mass matrix

$$
M_{\mathrm{eff}}=-M_{D} M_{R}^{-1} M_{D}^{T},
$$


with entries of the order $10^{-2} \mathrm{eV}$ in agreement with observation. The leptonic Dirac mass matrices $M_{\ell}$ and $M_{D}$, and the Majorana mass matrices $M_{R}$ and $M_{\text {eff }}$ are diagonalized by

$$
M_{\ell}=U_{\ell} M_{\ell}^{\text {diag }} U_{\ell^{\prime}}^{\dagger}, \quad M_{D}=U_{D} M_{D}^{\text {diag }} U_{D^{\prime}}^{\dagger}, \quad M_{R}=U_{R} M_{R}^{\text {diag }} U_{R}^{T}, \quad M_{\mathrm{eff}}=U_{\nu} M_{\mathrm{eff}}^{\text {diag }} U_{\nu}^{T},
$$

where $U_{\ell}, U_{\ell^{\prime}}, U_{D}, U_{D^{\prime}}, U_{R}$, and $U_{\nu}$, are unitary mixing matrices, whereas $M_{\ell}^{\text {diag }}, M_{D}^{\text {diag }}, M_{R}^{\text {diag }}$, and $M_{\mathrm{eff}}^{\text {diag }}$, are diagonal mass matrices with positive entries. The mass eigenvalues of the charged leptons and neutrinos are given by $M_{\ell}^{\text {diag }}=\operatorname{diag}\left(m_{e}, m_{\mu}, m_{\tau}\right)$ and $M_{\text {eff }}^{\text {diag }}=$ $\operatorname{diag}\left(m_{1}, m_{2}, m_{3}\right)$, where $m_{1}, m_{2}$, and $m_{3}$, are the first, second, and third neutrino mass eigenvalue. We can always write a mixing matrix $U_{x}$ as a product of the form

$$
U_{x}=D_{x} \widehat{U}_{x} K_{x}
$$

where $\widehat{U}_{x}$ is a CKM-like matrix that reads in the standard parameterization (we follow here throughout the conventions and definitions given in Ref. [28])

$$
\widehat{U}_{x}=\left(\begin{array}{ccc}
c_{12} c_{13} & s_{12} c_{13} & s_{13} e^{-\mathrm{i} \widehat{\delta}^{x}} \\
-s_{12} c_{23}-c_{12} s_{23} s_{13} e^{\mathrm{i} \widehat{\delta}^{x}} & c_{12} c_{23}-s_{12} s_{23} s_{13} e^{\mathrm{i} \widehat{\delta}^{x}} & s_{23} c_{13} \\
s_{12} s_{23}-c_{12} c_{23} s_{13} e^{\mathrm{i} \hat{\delta}^{x}} & -c_{12} s_{23}-s_{12} c_{23} s_{13} e^{\mathrm{i} \widehat{\delta}^{x}} & c_{23} c_{13}
\end{array}\right),
$$

with $s_{i j}=\sin \hat{\theta}_{i j}^{x}, c_{i j}=\cos \hat{\theta}_{i j}^{x}$, and $\hat{\theta}_{i j}^{x} \in\left\{\hat{\theta}_{12}^{x}, \hat{\theta}_{13}^{x}, \hat{\theta}_{23}^{x}\right\}$ lie all in the first quadrant, i.e., $\hat{\theta}_{i j} \in\left[0, \frac{\pi}{2}\right]$, and $\widehat{\delta}^{x} \in[0,2 \pi]$. In Eq. ([6]), $D_{x}$ and $K_{x}$ denote diagonal phase matrices that are $D_{x}=\operatorname{diag}\left(e^{\mathrm{i} \varphi_{1}^{x}}, e^{\mathrm{i} \varphi_{2}^{x}}, e^{\mathrm{i} \varphi_{3}^{x}}\right)$ and $K_{x}=\operatorname{diag}\left(e^{\mathrm{i} \alpha_{1}^{x}}, e^{\mathrm{i} \alpha_{2}^{x}}, 1\right)$, where the index $x$ runs over $x=\ell, \ell^{\prime}, D, D^{\prime}, R, \nu$. The phases in $D_{x}$ and $K_{x}$ are all in the range $[0,2 \pi]$. The PMNS matrix reads

$$
U_{\mathrm{PMNS}}=U_{\ell}^{\dagger} U_{\nu}=\widehat{U}_{\mathrm{PMNS}} K_{\mathrm{Maj}}
$$

where $\widehat{U}$ is a CKM-like matrix parameterized as in Eq. (7), and $K_{\text {Maj }}=\operatorname{diag}\left(e^{\mathrm{i} \phi_{1}}, e^{\mathrm{i} \phi_{2}}, 1\right)$ contains the Majorana phases $\phi_{1}$ and $\phi_{2}$. The CKM-like matrix $\widehat{U}_{\text {PMNS }}$ in Eq. (8) is described by the solar angle $\theta_{12}$, the reactor angle $\theta_{13}$, the atmospheric angle $\theta_{23}$, and the Dirac CPphase $\delta$, which we identify in the parameterization of Eq. (7) as $\hat{\theta}_{i j}^{x} \rightarrow \theta_{i j}$ and $\widehat{\delta}^{x} \rightarrow \delta$.

\section{Flavor Structure from $Z_{n}$ Symmetries}

Let us next extend the SM gauge group to $G_{\mathrm{SM}} \times G_{F}$, where $G_{F}$ is a flavor symmetry. We assume that $G_{F}$ is a direct product of discrete $Z_{n}$ symmetries, i.e.,

$$
G_{F}=Z_{n_{1}} \times Z_{n_{2}} \times \cdots \times Z_{n_{m}}
$$

where $m$ is the number of $Z_{n}$ factors and the $n_{k}(k=1,2, \ldots, m)$ may be different. We will denote by $\left|G_{F}\right|=\prod_{k=1}^{m} n_{k}$ the group order (i.e., the number of elements) of $G_{F}$. Under $G_{F}$, we assign to the leptons the charges

$$
e_{i}^{c} \sim\left(p_{1}^{i}, p_{2}^{i}, \ldots, p_{m}^{i}\right)=p^{i}, \quad \ell_{i} \sim\left(q_{1}^{i}, q_{2}^{i}, \ldots, q_{m}^{i}\right)=q^{i}, \quad \nu_{i}^{c} \sim\left(r_{1}^{i}, r_{2}^{i}, \ldots, r_{m}^{i}\right)=r^{i},
$$


where the $j$ th entry in each row vector denotes the $Z_{n_{j}}$ charge of the particle and $i=1,2,3$ is the generation index (see Sec. 2). In the following, we choose a convention where for each group $Z_{n_{k}}$ the charges are non-negative and lie in the range

$$
p_{k}^{i}, q_{k}^{i}, r_{k}^{i} \in\left\{0,1,2, \ldots, n_{k}-1\right\} .
$$

The sum $x_{k}+y_{k}$ of two $Z_{n_{k}}$ charges is equivalent with $x_{k}+y_{k} \bmod n_{k}$ and only determined modulo $n_{k}$. In order to spontaneously break the flavor symmetries, we assume for each factor $Z_{n_{k}}$ a single scalar flavon field $f_{n_{k}}$ that carries a charge -1 under $Z_{n_{k}}$ but is a singlet under all other $Z_{n_{j}}$ with $j \neq k$. Moreover, the $f_{n_{k}}$ are $G_{\mathrm{SM}}$ singlets.

When the $f_{n_{k}}$ acquire nonzero universal vacuum expectation values $\left\langle f_{n_{k}}\right\rangle=v$, nonrenormalizable lepton Yukawa couplings and mass terms of the form

$$
\begin{aligned}
\mathcal{L}_{\mathrm{Y}}= & -\left(\Pi_{k=1}^{m} \epsilon^{a_{i j}^{k}}\right)\left(Y_{\ell}^{\prime}\right)_{i j} H^{*} \ell_{i} e_{j}^{c}-\left(\Pi_{k=1}^{m} \epsilon^{b_{i j}^{k}}\right)\left(Y_{D}^{\prime}\right)_{i j} \mathrm{i} \sigma^{2} H \ell_{i} \nu_{j}^{c} \\
& -\frac{1}{2}\left(\Pi_{k=1}^{m} \epsilon^{c_{i j}^{k}}\right) M_{B-L}\left(Y_{R}^{\prime}\right)_{i j} \nu_{i}^{c} \nu_{j}^{c}+\text { h.c. }
\end{aligned}
$$

are generated by the Froggatt-Nielsen mechanism by integrating out heavy fermions with mass $M_{F}$, where $\epsilon=v / M_{F} \simeq \theta_{\mathrm{C}} \simeq 0.2$ is of the order of the Cabibbo angle, and

$$
\begin{aligned}
a_{i j}^{k} & =\min \left\{p_{i}^{k}+q_{j}^{k} \bmod n_{k},-p_{i}^{k}-q_{j}^{k} \bmod n_{k}\right\}, \\
b_{i j}^{k} & =\min \left\{q_{i}^{k}+r_{j}^{k} \bmod n_{k},-q_{i}^{k}-r_{j}^{k} \bmod n_{k}\right\}, \\
c_{i j}^{k} & =\min \left\{r_{i}^{k}+r_{j}^{k} \bmod n_{k},-r_{i}^{k}-r_{j}^{k} \bmod n_{k}\right\},
\end{aligned}
$$

whereas $Y_{\ell}^{\prime}, Y_{D}^{\prime}$, and $Y_{R}^{\prime}$, are dimensionless order unity Yukawa couplings. The modulo function $\bmod n_{k}$ in Eq. (13) is a consequence of the cyclic nature of the $Z_{n}$ symmetries, and the minimum takes into account that the higher dimension operators can be built from both $f_{n_{k}}$ and the complex conjugated fields $f_{n_{k}}^{*}$. The important point is that the $Z_{n_{k}}$ charges of the leptons determine a hierarchical pattern of the Yukawa coupling matrices and the right-handed Majorana neutrino mass matrix.

We define a "texture" as the matrix collecting the leading order products of $\epsilon$ for a certain Yukawa coupling or mass matrix, thereby ignoring the information on the order unity coefficients $Y_{\ell}^{\prime}, Y_{D}^{\prime}$, and $Y_{R}^{\prime}$. The lepton textures are therefore the $3 \times 3$ matrices with matrix elements approximating $\left(M_{\ell}\right)_{i j},\left(M_{D}\right)_{i j}$, and $\left(M_{R}\right)_{i j}$, as

$$
\left(M_{\ell}\right)_{i j} \approx \prod_{k=1}^{m} \epsilon^{a_{i j}^{k}}, \quad\left(M_{D}\right)_{i j} \approx \prod_{k=1}^{m} \epsilon^{b_{i j}^{k}}, \quad\left(M_{R}\right)_{i j} \approx \prod_{k=1}^{m} \epsilon^{c_{i j}^{k}}
$$

In what follows, we will, for a certain model call the set of three textures given in Eq. (14) a "texture set".

Note that in our models, the $Z_{n}$ flavor symmetries are global, but it might be important to gauge them to survive quantum gravity corrections [33]. The cancellation of anomalies for our symmetries could, e.g. be achieved by adding suitable extra matter fields which is, however, beyond the scope of this paper. 


\section{Scanning Approach}

In this section, we will describe how we select among the models introduced in Sec. 3 those which give nearly tribimaximal lepton mixing. First, we pick some flavor symmetry group $G_{F}$ as in Eq. (9) and assign to the three generations of leptons $e_{i}^{c}, \ell_{i}$, and $\nu_{i}^{c}$, all possible charge combinations under $G_{F}$. Then, we determine for each model from the charge assignment the corresponding lepton textures for $M_{\ell}, M_{D}$, and $M_{R}$, following Eq. (14). Next, to find models that can give a good fit to nearly tribimaximal lepton mixing, we compare the textures found in Eq. (14) with the list of 1981 representative texture sets given in Ref. [29]. In Ref. [29], we have, based on assumptions of EQLC, determined fits of the order one Yukawa couplings $Y_{\ell}^{\prime}, Y_{D}^{\prime}$, and $Y_{R}^{\prime}$, to reproduce nearly tribimaximal lepton mixing along with a charged lepton mass spectrum $m_{e}: m_{\mu}: m_{\tau}=\epsilon^{4}: \epsilon^{2}: 1$ and a normal neutrino mass hierarchy $m_{1}: m_{2}: m_{3}=\epsilon^{2}: \epsilon: 1$ in perfect agreement with current neutrino data (at the $3 \sigma$ confidence level (CL)).

In Ref. [29], the textures have been extracted in the basis where $U_{\ell^{\prime}}=\mathbb{1}$, i.e., where the rotations acting on the $e_{i}^{c}$ are zero. Although these rotations do not show up in the observables, they are important for formulating our explicit models using flavor symmetries. We therefore include now in our considerations textures of the charged leptons that have been extracted in bases where $U_{\ell^{\prime}}$ can be nontrivial, i.e., where we can have $U_{\ell^{\prime}} \neq \mathbb{1}$, thereby leading to a multitude of new explicit representations for the charged lepton textures. In complete analogy with Ref. [29], we assume for the mixing angles and phases entering the matrices $U_{\ell^{\prime}}$ all possible combinations that satisfy

$$
\theta_{12}^{\ell^{\prime}}, \theta_{13}^{\ell^{\prime}}, \theta_{23}^{\ell^{\prime}} \in\left\{0, \epsilon^{2}, \epsilon, \frac{\pi}{4}\right\}
$$

while the phases can take the values $\widehat{\delta}^{\ell^{\prime}}, \varphi_{1}^{\ell^{\prime}}, \varphi_{2}^{\ell^{\prime}} \in\{0, \pi\}$, whereas we can always choose $\varphi_{3}^{\ell^{\prime}}=\alpha_{1}^{\ell^{\prime}}=\alpha_{2}^{\ell^{\prime}}=0$ (for a definition of the notation, see Sec. 2). This applies the hypothesis of EQLC to the $e_{i}^{c}$ such that the left- and right-handed lepton sectors are now all treated on the same footing. As a consequence, after employing the texture reduction from Refs. [28, 29], we finally arrive at an enlarged "reference list" of 43278 qualitatively different lepton texture sets. We use this list as our reference to match onto flavor symmetry models for nearly tribimaximal lepton mixing. Any flavor charge assignment that yields textures contained in this reference list provides a valid model that allows an excellent fit to nearly tribimaximal lepton mixing 1

We can now impose extra assumptions on the properties of the textures in our reference list to search for interesting flavor symmetry models. For example, we will demand that none of the textures is completely anarchic (or democratic) and that the charged lepton textures contain at least one relative $\epsilon$-power $\geq 4$ (to have sufficient structure in the texture). This reduces the above reference list to a reduced list of 17772 distinct texture sets. In the next

\footnotetext{
${ }^{1}$ Note that in our reference list (after factoring out a possible overall power of $\epsilon$ ) we set an entry $\epsilon^{n}$ equal to zero when $n \geq 3$ (for neutrinos only) or when $n \geq 5$ (for charged leptons). This is different from Ref. 29, where such an entry is set to zero when $n \geq 3$ for both neutrinos and charged leptons. For specific flavor models, however, we will always show the actual suppression factor $\epsilon^{n}$, as predicted from the flavor symmetry.
} 
section, we will use this reduced reference list to perform the group space scan of flavor symmetries.

\section{Results of the Group Space Scan}

Let us now present the results of the group space scan for flavor symmetries that produce nearly tribimaximal lepton mixing. We assume throughout the models introduced in Sec. 3 and choose as flavor symmetries $G_{F}=\Pi_{k=1}^{m} Z_{n_{k}}$ for $m=1,2,3,4$. A complete scan has been performed for groups up to order 40 (for $m=1$ ), 45 (for $m=2$ ), 30 (for $m=3$ ), and 24 (for $m=4$ ), and valid models are selected as outlined in Sec. 4 by matching the textures generated by the flavor charge assignment onto the reduced reference list of 17772 non-anarchic texture sets. In total, we find in the scan 6021 such models that reproduce
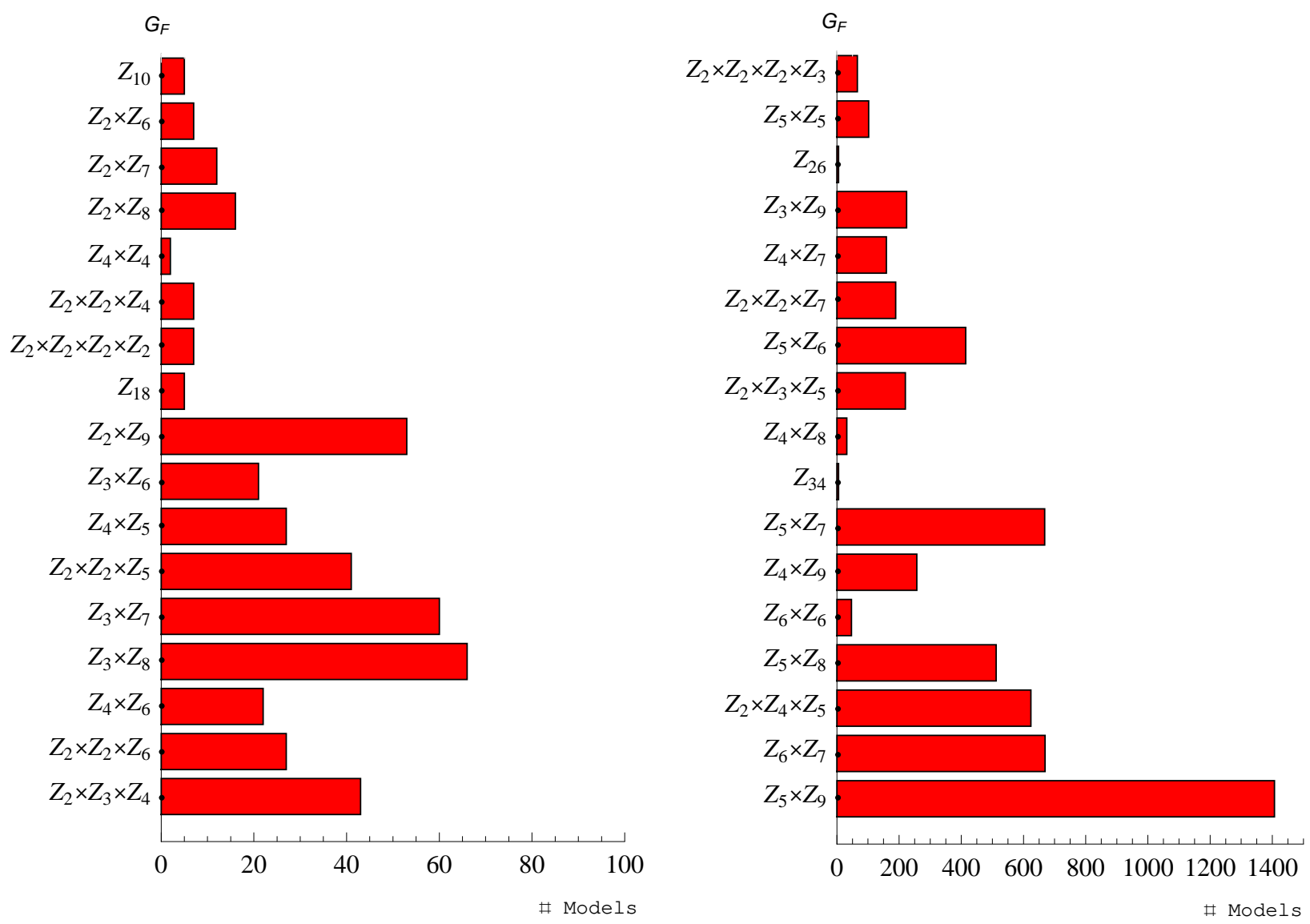

Figure 1: Number of flavor models leading to nearly tribimaximal lepton mixing as a function of the flavor group $G_{F}$ for increasing group order. In the left (right) panel we have $10 \leq$ $\left|G_{F}\right| \leq 24\left(24 \leq\left|G_{F}\right| \leq 45\right)$.

\footnotetext{
${ }^{2}$ For an application of related flavor groups see Ref. [34].
} 
2093 texture sets. The distribution of the selected models for the different groups $G_{F}$ is summarized in Fig. 1 $]^{3}$ All these models allow for an excellent fit to nearly tribimaximal neutrino mixing (at $3 \sigma \mathrm{CL}$ and most of them actually at $1 \sigma$ [29]) with a very small reactor angle $\theta_{13} \lesssim 1^{\circ}$ and the lepton mass ratios 4

$$
m_{e}: m_{\mu}: m_{\tau}=\epsilon^{4}: \epsilon^{2}: 1, \quad m_{1}: m_{2}: m_{3}=\epsilon^{2}: \epsilon: 1 \text {, }
$$

i.e., we have have a normal neutrino mass hierarchy with a ratio $\Delta m_{\odot}^{2} / \Delta m_{\text {atm }}^{2} \sim \epsilon^{2}$ of solar over atmospheric neutrino mass squared difference. Since the neutrinos have a normal mass hierarchy, renormalization group effects are negligible (for a discussion and references see Ref. [29]).

In Fig. 1, we can observe the trend that the number of valid models generally increases with the group order $\left|G_{F}\right|$ up to some periodic modulation. It is interesting to give a rough estimate on how large $G_{F}$ has to be in order to more or less reproduce an arbitrary texture set. For this purpose, note that (after factoring out a possible common overall factor $\epsilon^{n}$ ) we restrict ourselves in $M_{D}$ and $M_{R}$ to the matrix entries $\left\{0, \epsilon^{2}, \epsilon, 1\right\}$ and in $M_{\ell}$ to the entries $\left\{0, \epsilon^{4}, \epsilon^{3}, \epsilon^{2}, \epsilon, 1\right\}$, i.e., we have $4^{6+9} \cdot 6^{9}$ different possibilities for arbitrary texture sets. On the other hand, we have nine different charges per group, leading to $\left|G_{F}\right|^{9}$ different possibilities for the charge assignments in $G_{F}$ (see Eq. (10)). Note that we use $\left|G_{F}\right|$ as a figure of merit: The larger $\left|G_{F}\right|$, the more possibilities for the charge assignments we have. In order to reproduce any texture set, we estimate that the number of possibilities for the charges should exceed the number of possibilities for the texture sets, i.e.,

$$
\left|G_{F}\right| \gtrsim 4^{\frac{15}{9}} \cdot 6 \simeq 60
$$

That means that, for instance, four $Z_{n}$ factors with moderate $n_{k}$, such as $Z_{2} \times Z_{3} \times Z_{4} \times Z_{5}$ should be sufficient, or two $Z_{n}$ factors with high $n_{k}$, such as $Z_{7}$ and higher. In Fig. 1, we have much less possibilities on the l.h.s. where $10 \leq\left|G_{F}\right| \leq 24$, while we have on the r.h.s. $24 \leq\left|G_{F}\right| \leq 45$. In fact, Fig. 1 seems to suggest that $G_{F}=Z_{5} \times Z_{9}$ with $\left|G_{F}\right|=45$ is entering the regime estimated in Eq. (17).

We have checked that for the 6021 valid models practically all (i.e., more than 99.9\%) of the Yukawa coupling matrix elements of $Y_{\ell}^{\prime}, Y_{D}^{\prime}$, and $Y_{R}^{\prime}$, (see Eq. (12)) lie in the interval between $\epsilon$ and $1 / \epsilon$. With respect to the expansion parameter $\epsilon$ of our models, these matrix elements can therefore indeed be viewed as order one coefficients.

Out of our set of 6021 valid models, let us now consider a few examples. In Tab. 1, we show 22 explicit models by listing the flavor group with complete flavor charge assignment and the resulting textures for $M_{\ell}, M_{D}$, and $M_{R}$. The rough guideline for choosing these 22 models was to give one example for each texture set previously identified in EQLC [29], with distinct $M_{R}$ and a charged lepton mass spectrum as in Eq. (16). The complete information on the corresponding mass and mixing parameters of the 22 models is summarized in Table 2 in the Appendix.

Table 2 demonstrates that our models are very general in the sense that they can exhibit maximal mixings in the charged lepton and/or the neutrino sector, and that the left- and/or

\footnotetext{
${ }^{3}$ Additionally, we have also included the number of valid models for $G_{F}=Z_{2} \times Z_{4} \times Z_{5}$.

${ }^{4}$ We are interested in an $S U(5)$ compatible fit.
} 
right-handed neutrinos can mix maximally. In addition, we can have maximal mixings in all sectors not only between the 2nd and 3rd generation but also between the 1st and the 2nd as well as between the 1st and the 3rd generation.

All 22 models in Tables 1 and 2 lead to the following PMNS mixing angles in the range

$$
34^{\circ} \lesssim \theta_{12} \lesssim 39^{\circ}, \quad \theta_{13} \lesssim 1^{\circ}, \quad \theta_{23} \approx 52^{\circ}
$$

in agreement with neutrino oscillation data (at $3 \sigma \mathrm{CL}$ ). The models are thus characterized by a very small reactor angle close to zero, and a significant deviation of about $+5^{\circ}$ from maximal atmospheric mixing.

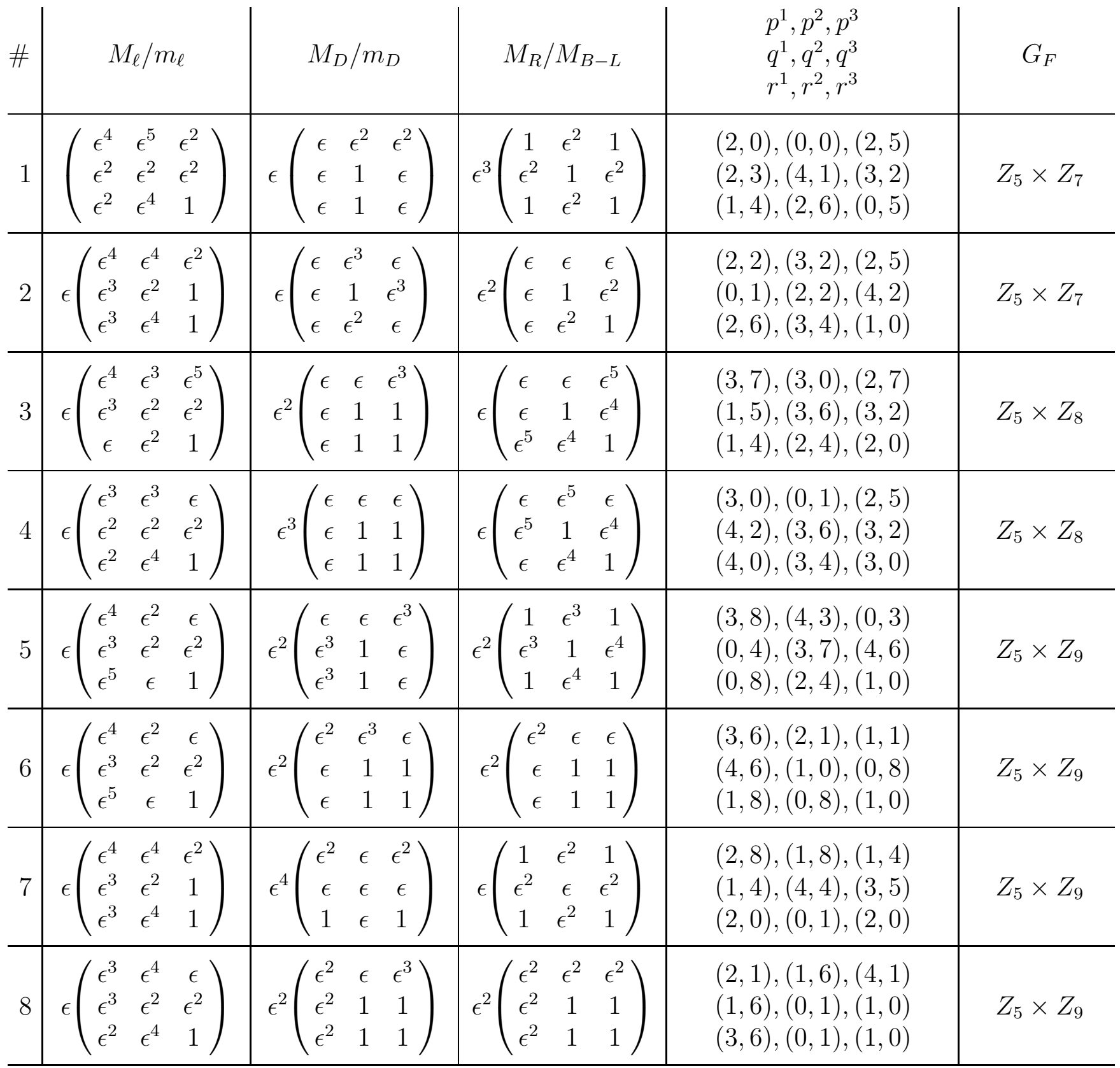




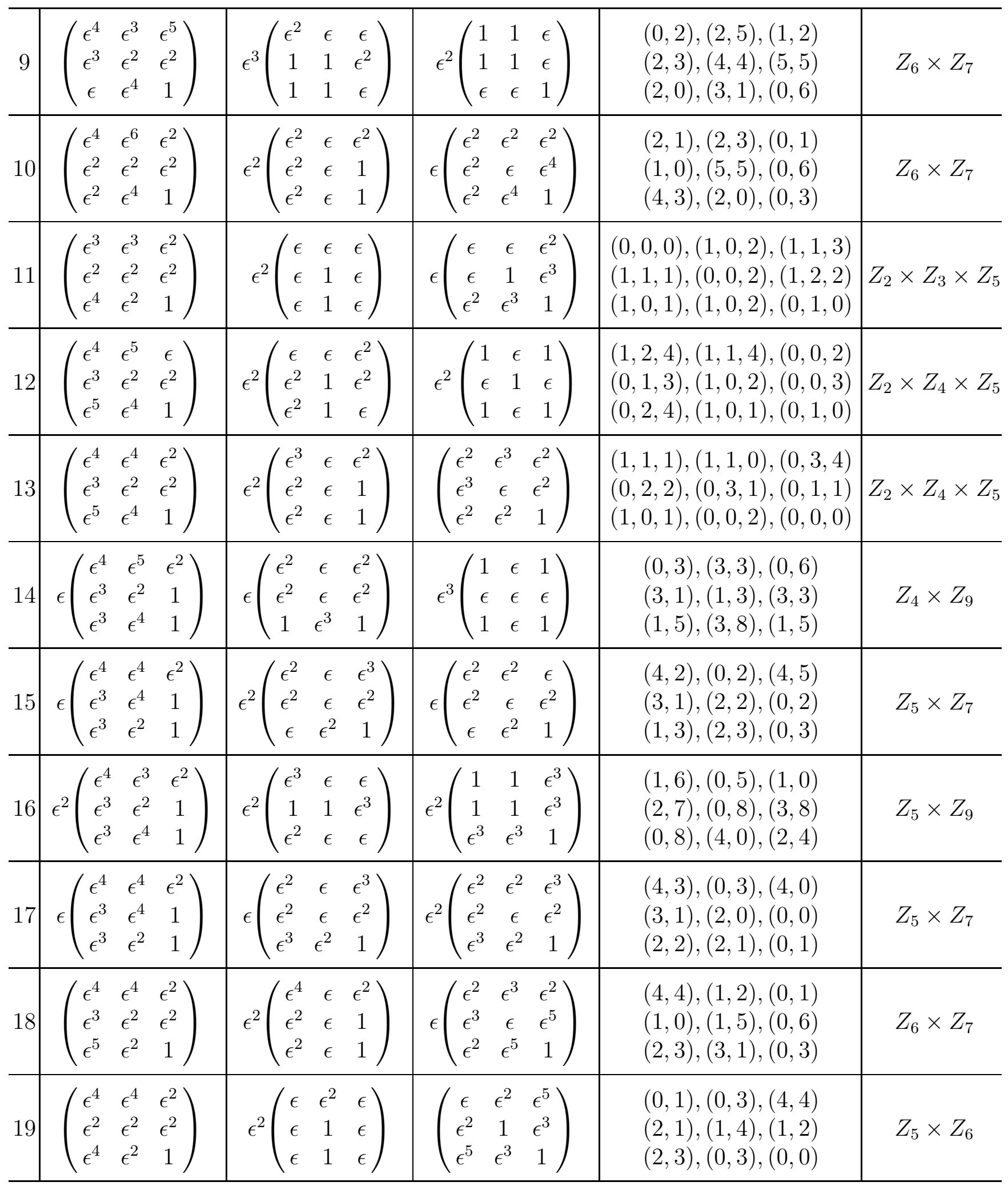




\begin{tabular}{l|c|c|c|c|c|c}
\hline 20 & $\left(\begin{array}{ccc}\epsilon^{4} & \epsilon^{5} & \epsilon^{2} \\
\epsilon^{2} & \epsilon^{2} & \epsilon^{2} \\
\epsilon^{4} & \epsilon^{4} & 1\end{array}\right)$ & $\epsilon\left(\begin{array}{ccc}\epsilon^{2} & \epsilon & \epsilon^{2} \\
\epsilon^{2} & \epsilon & 1 \\
\epsilon^{2} & \epsilon & 1\end{array}\right)$ & $\epsilon\left(\begin{array}{ccc}\epsilon^{2} & \epsilon^{2} & \epsilon^{2} \\
\epsilon^{2} & \epsilon & \epsilon^{2} \\
\epsilon^{2} & \epsilon^{2} & 1\end{array}\right)$ & $\begin{array}{l}(2,0),(3,5),(1,3) \\
(0,4),(3,2),(4,3) \\
(2,1),(0,4),(2,3)\end{array}$ & $Z_{5} \times Z_{6}$ \\
\hline 21 & $\left(\begin{array}{ccc}\epsilon^{4} & \epsilon^{5} & \epsilon^{2} \\
\epsilon^{2} & \epsilon^{2} & \epsilon^{2} \\
\epsilon^{4} & \epsilon^{4} & 1\end{array}\right)$ & $\epsilon\left(\begin{array}{ccc}\epsilon & \epsilon^{2} & \epsilon \\
\epsilon & 1 & \epsilon \\
\epsilon^{3} & 1 & \epsilon\end{array}\right)$ & $\epsilon^{2}\left(\begin{array}{ccc}\epsilon & \epsilon^{2} & \epsilon \\
\epsilon^{2} & 1 & \epsilon^{3} \\
\epsilon & \epsilon^{3} & 1\end{array}\right)$ & $\begin{array}{l}(3,4),(4,4),(1,2) \\
(3,5),(4,2),(4,4) \\
(2,5),(1,3),(1,0)\end{array}$ & $Z_{5} \times Z_{6}$ \\
\hline 22 & $\left(\begin{array}{ccc}\epsilon^{4} & \epsilon^{3} & \epsilon^{2} \\
\epsilon^{2} & \epsilon^{2} & \epsilon^{3} \\
\epsilon^{5} & \epsilon & 1\end{array}\right)$ & $\epsilon^{2}\left(\begin{array}{ccc}\epsilon^{2} & \epsilon & \epsilon^{2} \\
1 & \epsilon & 1 \\
1 & \epsilon^{3} & 1\end{array}\right)$ & $\epsilon\left(\begin{array}{ccc}1 & \epsilon^{3} & 1 \\
\epsilon^{3} & \epsilon & \epsilon^{3} \\
1 & \epsilon^{3} & 1\end{array}\right)$ & $\begin{array}{l}(2,6),(0,0),(0,1) \\
(0,6),(1,1),(0,8) \\
(1,0),(2,5),(1,0)\end{array}$ & $Z_{3} \times Z_{9}$ \\
\hline
\end{tabular}

Table 1: A list of 22 flavor models for nearly tribimaximal lepton mixing. Shown are the explicit flavor charges under the flavor symmetry group $G_{F}$ and the resulting textures. Possible common overall suppression factors have been factored out of the textures.

Some of the models in Table 1 allow to extract very easily new sum rules for the leptonic mixing angles using an expansion in $\epsilon$. For example, we find for model \#6 the sum rules

$$
\theta_{12}=\frac{\pi}{4}-\frac{\epsilon}{\sqrt{2}}-\frac{\epsilon^{2}}{4}, \quad \theta_{13}=\left(\frac{1}{\sqrt{2}}-\frac{1}{2}\right) \epsilon^{2}, \quad \theta_{23}=\frac{\pi}{4}+\frac{\epsilon}{\sqrt{2}}+\left(\frac{1}{\sqrt{2}}-\frac{5}{4}\right) \epsilon^{2},
$$

while model \#8 exhibits the relations

$$
\theta_{12}=\frac{\pi}{4}-\frac{\epsilon}{\sqrt{2}}+\left(\frac{1}{\sqrt{2}}-\frac{9}{4}\right) \epsilon^{2}, \quad \theta_{13}=\frac{\epsilon^{2}}{2}, \quad \theta_{23}=\frac{\pi}{4}+\frac{\epsilon}{\sqrt{2}}+\left(\frac{1}{\sqrt{2}}-\frac{5}{4}\right) \epsilon^{2} .
$$

For both the models \#6 and \#8, the solar angle satisfies the well-known QLC relation $\theta_{12} \approx \frac{\pi}{4}-\theta_{\mathrm{C}} / \sqrt{2}$. The reactor angle, on the other hand, becomes for these two models small due to an apparent suppression by a factor $\sim \theta_{\mathrm{C}}^{2}$. Note that a similar suppression of the reactor angle has been found for the model in Ref. [35]. The atmospheric angle follows in both models the new sum rule $\theta_{23} \approx \frac{\pi}{4}+\theta_{\mathrm{C}} / \sqrt{2}$, which predicts a deviation from maximal mixing by an amount of approximately $\theta_{\mathrm{C}} / \sqrt{2}$. This prediction makes these models testable in future neutrino oscillation experiments. For example, the deviation from maximal mixing can be established at $3 \sigma \mathrm{CL}$ by the $\mathrm{T} 2 \mathrm{~K}$ or $\mathrm{NO} \nu$ a experiments [36. In addition, one can measure the sign of the deviation from maximal mixing (the octant) with a neutrino factory at $3 \sigma$ CL for $\sin ^{2} 2 \theta_{13} \gtrsim 10^{-2.5}$ or at $90 \%$ CL otherwise [37].

The relations for $\theta_{13}$ in Eqs. (19) and (20), however, are not stable under variations of the Yukawa couplings and must therefore be a result of exact cancellations between large contributions from different sectors. To render these relations for $\theta_{13}$ natural, it might therefore be necessary to extend these models by non-Abelian discrete symmetries, which shall be addressed elsewhere [38]. 
Let us therefore have a look at model \#5, which has the sum rules

$$
\theta_{12}=\frac{\pi}{4}-\frac{\epsilon}{\sqrt{2}}-\frac{\epsilon^{2}}{4}, \quad \theta_{13}=\mathcal{O}\left(\epsilon^{3}\right), \quad \theta_{23}=\frac{\pi}{4}+\frac{\epsilon}{\sqrt{2}}-\frac{3}{4} \epsilon^{2} .
$$

The sum rules for $\theta_{12}$ and $\theta_{23}$ are to leading order just as in the two previous examples. The relation for $\theta_{13}$, however, is different from that in Eqs. (19) and (20): Now, $\theta_{13}$ is very small due to a suppression by a factor $\sim \theta_{\mathrm{C}}^{3}$. Unlike for models $\# 6$ and $\# 8$, the relation for $\theta_{13}$ in Eq. (21) is stable under variations of the Yukawa couplings. This means, by varying the Yukawa couplings, $\theta_{13}$ picks up only a small relative correction that is suppressed by a factor $\sim \theta_{\mathrm{C}}^{3}$. The stability of the relation $\theta_{13} \simeq \mathcal{O}\left(\epsilon^{3}\right)$ under $1 \%$ variations of the order one
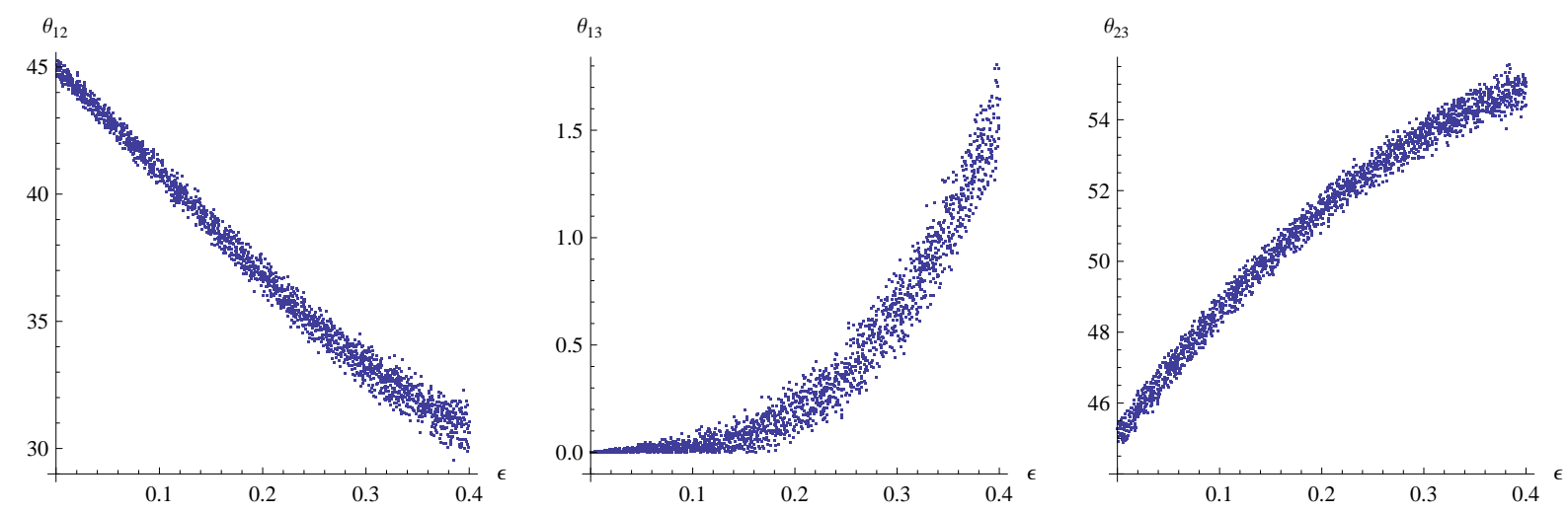

Figure 2: Solar (left), reactor (middle), and atmospheric (right) mixing angles (in degrees), for model \#5 in Table 1 as a function of the expansion parameter $\epsilon$. The points correspond to a random variation of the order one Yukawa couplings in $Y_{\ell}^{\prime}, Y_{D}^{\prime}$, and $Y_{R}^{\prime}$, by about $1 \%$. The plot in the middle shows that the relation $\theta_{13} \simeq \mathcal{O}\left(\epsilon^{3}\right)$ in Eq. (21) is not due to an accidental cancellation between large mixing angles.

Yukawa couplings is shown in Fig. 2. The relations for $\theta_{12}$ and $\theta_{23}$ are in all three above examples in Eqs. (19), (20), and (21), in this sense anyway stable under variations of the Yukawa couplings.

In analogy with the $Z_{n}$ groups, we have also performed a scan of $U(1)$ symmetries. In order to see whether the cyclic character of the discrete groups is a relevant feature in the construction of valid models, we have compared single $Z_{n}$ groups $G_{F}=Z_{n_{1}}$ up to $\left|G_{F}\right|=40$ and product groups $G_{F}=Z_{n_{1}} \times Z_{n_{2}}$ up to $\left|G_{F}\right|=18$ with corresponding Abelian groups, i.e., with $U(1)$ (for $G_{F}=Z_{n_{k}}$ ) and $U(1) \times U(1)$ (for $G_{F}=Z_{n_{1}} \times Z_{n_{2}}$ ) by letting (the absolute value of) the respective individual $U(1)$ charges vary in the whole range $0,1, \ldots, n_{k}-1$. Thereby, we have identified 129 models for the cyclic groups and 24 models using $U(1)$ symmetries. All of the $24 U(1)$ models correspond to the group $G_{F}=Z_{3} \times Z_{6}$, for which we have obtained in total 21 models. However, even though the $U(1)$ models produce textures that are very similar to those of model \#6 in Table 1 (with varying $M_{\ell}$ and varying first row in $M_{D}$ ), the mass scales $m_{D}$ and $M_{R}$ get in these examples highly suppressed by factors $\lesssim \epsilon^{8}$. This analysis already indicates that the cyclic character of the flavor groups may be essential for facilitating the construction of realistic flavor models. 


\section{Summary and Conclusions}

In this paper, we have constructed several thousand explicit flavor models for nearly tribimaximal lepton mixing from products of $Z_{n}$ flavor symmetries. In our models, small neutrino masses emerge only from the canonical type-I seesaw mechanism. Upon flavor symmetry breaking, the Froggatt-Nielsen mechanism produces hierarchical Yukawa coupling and mass matrix textures of the leptons. These textures are parameterized by powers of a single small symmetry breaking parameter $\epsilon \simeq \theta_{\mathrm{C}} \simeq 0.2$ that is of the order of the Cabibbo angle $\theta_{\mathrm{C}}$ and arises from integrating out heavy Froggatt-Nielsen messenger fermions.

All flavor models realize the assumptions of EQLC and yield an excellent fit to nearly tribimaximal neutrino mixing with a very small reactor angle $\theta_{13} \approx 0$. Moreover, they lead to the hierarchical charged lepton mass spectrum as well as to normal hierarchical neutrino masses. In our analysis, we have restricted ourselves to the most general CP-conserving case of real lepton mass matrices. We have performed a systematic scan of the group space for groups with up to four $Z_{n}$ factors and a maximum group order of 45 . As a consequence, we have found 6021 models that reproduce 2093 distinct texture sets.

A characteristic property of our flavor models is that large leptonic mixings can come from the charged leptons and/or neutrinos. In the neutrino sector, maximal mixings can arise in both the Dirac mass matrix or in the heavy right-handed Majorana mass matrix. Generally, we can have maximal mixings between any two generations in any lepton sector.

Among several explicit models, we have found a model that predicts a new relation $\theta_{13}=\mathcal{O}\left(\theta_{\mathrm{C}}^{3}\right)$, for the reactor angle. Moreover, we found models that all satisfy a new sum rule $\theta_{23} \approx \frac{\pi}{4}+\theta_{\mathrm{C}} / \sqrt{2}$ for the atmospheric mixing angle, which makes these models testable in future neutrino oscillation experiments such as the $\mathrm{T} 2 \mathrm{~K}$ and $\mathrm{NO} \nu$ a experiments or at a neutrino factory.

We wish to point out that in this paper we have established a connection between a model building top-down approach using flavor symmetries and the phenomenological bottom-up approach of Ref. [29]. While Ref. [29] deals with the extraction of viable lepton mass textures that are in agreement with observation, the current work successfully matches the textures onto explicit flavor models, where the textures are predicted from flavor symmetries and their breaking.

We believe that it would be interesting to study our sample of flavor models with respect to further model building aspects, anomaly cancellation, the inclusion of CP-violating phases, as well as in view of lepton flavor violation or leptogenesis.

\section{Appendix}

In this Appendix, we list the complete information on the mass and mixing parameters of the matrices that are generated by the models in Table 1, Here, "\#" labels in both tables the same model. The data in Table 2 allows to fully reconstruct the exact form of the mass matrices of the 22 models following the notation of Sec. 2 (for further detailed examples on such reconstructions, see also Ref. [29]). 


\begin{tabular}{|c|c|c|c|c|c|c|}
\hline$\#$ & $\begin{array}{c}m_{i}^{D} / m_{D} \\
m_{i}^{R} / M_{B-L}\end{array}$ & $\begin{array}{c}\left(\theta_{12}^{\ell}, \theta_{13}^{\ell}, \theta_{23}^{\ell}\right) \\
\left(\delta^{\ell}, \alpha_{1}^{\ell}, \alpha_{2}^{\ell}\right) \\
\end{array}$ & $\begin{array}{l}\left(\theta_{12}^{\ell^{\prime}}, \theta_{13}^{\ell^{\prime}}, \theta_{23}^{\ell^{\prime}}\right) \\
\left(\delta^{\ell^{\prime}}, \alpha_{1}^{\ell^{\prime}}, \alpha_{2}^{\ell^{\prime}}\right) \\
\end{array}$ & $\begin{array}{c}\left(\theta_{12}^{D}, \theta_{13}^{D}, \theta_{23}^{D}\right) \\
\left(\delta^{D}, \varphi_{1}^{D}, \varphi_{2}^{D}, \varphi_{3}^{D}\right) \\
\end{array}$ & $\begin{array}{l}\left(\theta_{12}^{D^{\prime}}, \theta_{13}^{D^{\prime}}, \theta_{23}^{D^{\prime}}\right) \\
\left(\delta^{D^{\prime}}, \alpha_{1}^{D^{\prime}}, \alpha_{2}^{D^{\prime}}\right) \\
\end{array}$ & $\begin{array}{c}\left(\theta_{12}^{R}, \theta_{13}^{R}, \theta_{23}^{R}\right) \\
\left(\delta^{R}, \varphi_{1}^{R}, \varphi_{2}^{R}, \varphi_{3}^{R}\right)\end{array}$ \\
\hline 1 & $\begin{array}{l}(\epsilon, 1, \epsilon) \\
(\epsilon, 1,1)\end{array}$ & $\begin{array}{c}\left(\epsilon^{2}, \epsilon^{2}, \epsilon^{2}\right) \\
(\pi, \pi, \pi)\end{array}$ & $\begin{array}{l}\left(\frac{\pi}{4}, \epsilon^{2}, 0\right) \\
(0,0,0)\end{array}$ & $\begin{array}{l}\left(0, \epsilon^{2}, \frac{\pi}{4}\right) \\
(0,0,0,0)\end{array}$ & $\begin{array}{l}\left(\epsilon, \epsilon, \epsilon^{2}\right) \\
(0,0,0)\end{array}$ & $\begin{array}{l}\left(\epsilon^{2}, \frac{\pi}{4}, \epsilon^{2}\right) \\
(\pi, 0, \pi, 0)\end{array}$ \\
\hline 2 & $\begin{array}{l}(\epsilon, 1, \epsilon) \\
(\epsilon, 1,1)\end{array}$ & $\begin{array}{c}\left(\epsilon^{2}, \epsilon^{2}, \frac{\pi}{4}\right) \\
(0,0,0)\end{array}$ & $\begin{array}{c}\left(\epsilon, 0, \epsilon^{2}\right) \\
(0,0,0)\end{array}$ & $\begin{array}{l}\left(\epsilon^{2}, \frac{\pi}{4}, \epsilon^{2}\right) \\
(0,0,0,0)\end{array}$ & $\begin{array}{l}\left(\epsilon, \epsilon^{2}, 0\right) \\
(0, \pi, \pi)\end{array}$ & $\begin{array}{c}\left(\epsilon, 0, \frac{\pi}{4}\right) \\
(0,0,0,0)\end{array}$ \\
\hline 3 & $\begin{array}{l}(\epsilon, 1, \epsilon) \\
(\epsilon, 1,1) \\
\end{array}$ & $\begin{array}{l}\left(\epsilon, 0, \epsilon^{2}\right) \\
(0,0,0) \\
\end{array}$ & $\begin{array}{l}\left(\epsilon, \epsilon, \epsilon^{2}\right) \\
(0,0,0) \\
\end{array}$ & $\begin{array}{c}\left(\epsilon, \frac{\pi}{4}, \frac{\pi}{4}\right) \\
(\pi, 0,0, \pi)\end{array}$ & $\begin{array}{l}\left(\epsilon, \epsilon, \frac{\pi}{4}\right) \\
(0, \pi, \pi) \\
\end{array}$ & $\begin{array}{c}\left(\epsilon, \epsilon, \frac{\pi}{4}\right) \\
(0,0,0, \pi) \\
\end{array}$ \\
\hline 4 & $\begin{array}{l}(\epsilon, \epsilon, 1) \\
(\epsilon, 1,1) \\
\end{array}$ & $\begin{array}{l}\left(\epsilon, \epsilon, \epsilon^{2}\right) \\
(0, \pi, \pi) \\
\end{array}$ & $\begin{array}{l}\left(\frac{\pi}{4}, \epsilon^{2}, 0\right) \\
(0,0,0)\end{array}$ & $\begin{array}{l}\left(\frac{\pi}{4}, \epsilon^{2}, \frac{\pi}{4}\right) \\
(0,0, \pi, 0)\end{array}$ & $\begin{array}{c}\left(0, \epsilon^{2}, \frac{\pi}{4}\right) \\
(0,0,0)\end{array}$ & $\begin{array}{c}\left(0, \epsilon, \epsilon^{2}\right) \\
(0,0, \pi, 0)\end{array}$ \\
\hline 5 & $\begin{array}{l}(\epsilon, 1, \epsilon) \\
(\epsilon, 1,1)\end{array}$ & $\begin{array}{r}\left(0, \epsilon, \epsilon^{2}\right) \\
(0, \pi, 0) \\
\end{array}$ & $\begin{array}{l}(\epsilon, 0, \epsilon) \\
(0,0,0) \\
\end{array}$ & $\begin{array}{c}\left(\epsilon, \frac{\pi}{4}, \frac{\pi}{4}\right) \\
(\pi, 0,0, \pi)\end{array}$ & $\begin{array}{c}\left(\epsilon^{2}, \frac{\pi}{4}, \epsilon^{2}\right) \\
(0, \pi, \pi)\end{array}$ & $\begin{array}{c}\left(0, \frac{\pi}{4}, 0\right) \\
(0,0,0, \pi)\end{array}$ \\
\hline 6 & $\begin{array}{l}\left(\epsilon^{2}, 1, \epsilon\right) \\
\left(\epsilon^{2}, \epsilon, 1\right) \\
\end{array}$ & $\begin{array}{l}\left(0, \epsilon, \epsilon^{2}\right) \\
(0, \pi, 0) \\
\end{array}$ & $\begin{array}{l}(\epsilon, 0, \epsilon) \\
(0,0,0) \\
\end{array}$ & $\begin{array}{c}\left(\epsilon, \frac{\pi}{4}, \frac{\pi}{4}\right) \\
(\pi, 0,0, \pi)\end{array}$ & $\begin{array}{l}\left(\epsilon, \epsilon, \frac{\pi}{4}\right) \\
(\pi, 0,0) \\
\end{array}$ & $\begin{array}{c}\left(\epsilon, \epsilon, \frac{\pi}{4}\right) \\
(\pi, 0, \pi, 0)\end{array}$ \\
\hline 7 & $\begin{array}{l}\left(\epsilon^{2}, \epsilon, 1\right) \\
\left(\epsilon^{2}, \epsilon, 1\right)\end{array}$ & $\begin{array}{l}\left(0, \epsilon^{2}, \frac{\pi}{4}\right) \\
(0, \pi, \pi)\end{array}$ & $\begin{array}{r}\left(\epsilon, 0, \epsilon^{2}\right) \\
(0,0, \pi)\end{array}$ & $\begin{array}{c}\left(\frac{\pi}{4}, \epsilon^{2}, \epsilon\right) \\
(0,0,0, \pi)\end{array}$ & $\begin{array}{l}\left(0, \frac{\pi}{4}, \epsilon\right) \\
(0,0, \pi)\end{array}$ & $\begin{array}{l}\left(\epsilon^{2}, \frac{\pi}{4}, \epsilon^{2}\right) \\
(\pi, 0, \pi, \pi)\end{array}$ \\
\hline 8 & $\begin{array}{l}\left(\epsilon^{2}, 1, \epsilon\right) \\
\left(\epsilon^{2}, \epsilon, 1\right)\end{array}$ & $\begin{array}{c}\left(\epsilon^{2}, \epsilon, \epsilon^{2}\right) \\
(0, \pi, 0)\end{array}$ & $\begin{array}{c}\left(\epsilon, \epsilon^{2}, 0\right) \\
(0,0,0)\end{array}$ & $\begin{array}{c}\left(\epsilon, \frac{\pi}{4}, \frac{\pi}{4}\right) \\
(\pi, 0,0, \pi)\end{array}$ & $\begin{array}{c}\left(0, \epsilon^{2}, \frac{\pi}{4}\right) \\
(0,0, \pi)\end{array}$ & $\begin{array}{c}\left(\epsilon^{2}, \epsilon^{2}, \frac{\pi}{4}\right) \\
(\pi, 0, \pi, 0)\end{array}$ \\
\hline 9 & $\begin{array}{l}(\epsilon, 1, \epsilon) \\
(\epsilon, 1,1)\end{array}$ & $\begin{array}{c}\left(\epsilon, 0, \epsilon^{2}\right) \\
(0,0,0)\end{array}$ & $\begin{array}{l}(\epsilon, \epsilon, 0) \\
(0,0,0)\end{array}$ & $\begin{array}{c}\left(\epsilon, \frac{\pi}{4}, \frac{\pi}{4}\right) \\
(\pi, 0,0, \pi)\end{array}$ & $\begin{array}{l}\left(\frac{\pi}{4}, \epsilon, \epsilon^{2}\right) \\
(\pi, 0, \pi)\end{array}$ & $\begin{array}{c}\left(\frac{\pi}{4}, \epsilon, 0\right) \\
(0,0,0, \pi)\end{array}$ \\
\hline 10 & $\begin{array}{l}\left(\epsilon^{2}, \epsilon, 1\right) \\
\left(\epsilon^{2}, \epsilon, 1\right)\end{array}$ & $\begin{array}{c}\left(\epsilon^{2}, \epsilon^{2}, \epsilon^{2}\right) \\
(\pi, \pi, \pi)\end{array}$ & $\begin{array}{c}\left(\frac{\pi}{4}, \epsilon^{2}, 0\right) \\
(0,0,0)\end{array}$ & $\begin{array}{l}\left(\frac{\pi}{4}, \epsilon^{2}, \frac{\pi}{4}\right) \\
(0,0,0, \pi)\end{array}$ & $\begin{array}{c}\left(\epsilon^{2}, 0, \epsilon^{2}\right) \\
(0,0,0)\end{array}$ & $\begin{array}{c}\left(\epsilon, \epsilon^{2}, 0\right) \\
(0,0,0,0)\end{array}$ \\
\hline 11 & $\begin{array}{l}(\epsilon, 1, \epsilon) \\
(\epsilon, 1,1)\end{array}$ & $\begin{array}{c}\left(\epsilon, \epsilon^{2}, \epsilon^{2}\right) \\
(\pi, 0,0)\end{array}$ & $\begin{array}{c}\left(\frac{\pi}{4}, 0, \epsilon^{2}\right) \\
(0,0,0)\end{array}$ & $\begin{array}{c}\left(\epsilon, \frac{\pi}{4}, \frac{\pi}{4}\right) \\
(\pi, 0,0, \pi)\end{array}$ & $\begin{array}{l}\left(\epsilon, \epsilon^{2}, \epsilon\right) \\
(\pi, 0,0)\end{array}$ & $\begin{array}{c}\left(\epsilon, \epsilon^{2}, \epsilon\right) \\
(\pi, 0,0,0)\end{array}$ \\
\hline 12 & $\begin{array}{l}(\epsilon, 1, \epsilon) \\
(\epsilon, 1,1) \\
\end{array}$ & $\begin{array}{r}\left(0, \epsilon, \epsilon^{2}\right) \\
(0, \pi, 0) \\
\end{array}$ & $\begin{array}{l}(\epsilon, 0,0) \\
(0,0,0) \\
\end{array}$ & $\begin{array}{c}\left(\epsilon, \frac{\pi}{4}, \frac{\pi}{4}\right) \\
(\pi, 0,0, \pi)\end{array}$ & $\begin{array}{c}\left(\epsilon^{2}, \frac{\pi}{4}, \epsilon\right) \\
(0, \pi, 0) \\
\end{array}$ & $\begin{array}{c}\left(\epsilon, \frac{\pi}{4}, \epsilon^{2}\right) \\
(\pi, 0, \pi, 0) \\
\end{array}$ \\
\hline 13 & $\begin{array}{l}\left(\epsilon^{2}, \epsilon, 1\right) \\
\left(\epsilon^{2}, \epsilon, 1\right)\end{array}$ & $\begin{array}{c}\left(\epsilon^{2}, \epsilon^{2}, \epsilon^{2}\right) \\
(\pi, 0, \pi) \\
\end{array}$ & $\begin{array}{l}(\epsilon, 0,0) \\
(0,0,0) \\
\end{array}$ & $\begin{array}{c}\left(\frac{\pi}{4}, \epsilon^{2}, \frac{\pi}{4}\right) \\
(\pi, 0, \pi, 0)\end{array}$ & $\begin{array}{c}\left(\epsilon, \epsilon^{2}, 0\right) \\
(0, \pi, 0)\end{array}$ & $\begin{array}{c}\left(0, \epsilon^{2}, \epsilon^{2}\right) \\
(0,0, \pi, \pi)\end{array}$ \\
\hline 14 & $\begin{array}{l}\left(\epsilon^{2}, \epsilon, 1\right) \\
\left(\epsilon^{2}, \epsilon, 1\right)\end{array}$ & $\begin{array}{c}\left(\epsilon^{2}, \epsilon^{2}, \frac{\pi}{4}\right) \\
(\pi, \pi, \pi)\end{array}$ & $\begin{array}{l}\left(\epsilon, 0, \epsilon^{2}\right) \\
(0,0, \pi)\end{array}$ & $\begin{array}{c}\left(\frac{\pi}{4}, \epsilon^{2}, 0\right) \\
(0,0,0,0)\end{array}$ & $\begin{array}{c}\left(\epsilon^{2}, \frac{\pi}{4}, 0\right) \\
(0,0, \pi)\end{array}$ & $\begin{array}{c}\left(\epsilon^{2}, \frac{\pi}{4}, \epsilon\right) \\
(0,0, \pi, 0)\end{array}$ \\
\hline 15 & $\begin{array}{l}\left(\epsilon^{2}, \epsilon, 1\right) \\
\left(\epsilon^{2}, \epsilon, 1\right)\end{array}$ & $\begin{array}{c}\left(\epsilon^{2}, \epsilon^{2}, \frac{\pi}{4}\right) \\
(\pi, \pi, \pi)\end{array}$ & $\begin{array}{c}\left(\epsilon, 0, \epsilon^{2}\right) \\
(0,0,0)\end{array}$ & $\begin{array}{c}\left(\frac{\pi}{4}, 0, \epsilon^{2}\right) \\
(0,0,0, \pi)\end{array}$ & $\begin{array}{c}\left(\epsilon^{2}, \epsilon, \epsilon^{2}\right) \\
(0,0,0)\end{array}$ & $\begin{array}{c}\left(\epsilon, \epsilon, \epsilon^{2}\right) \\
(\pi, 0,0, \pi)\end{array}$ \\
\hline 16 & $\begin{array}{l}(\epsilon, 1, \epsilon) \\
(\epsilon, 1,1) \\
\end{array}$ & $\begin{array}{c}\left(\epsilon, \epsilon^{2}, \frac{\pi}{4}\right) \\
(\pi, 0,0) \\
\end{array}$ & $\begin{array}{c}\left(\epsilon, 0, \epsilon^{2}\right) \\
(0,0,0) \\
\end{array}$ & $\begin{array}{c}\left(\epsilon, \frac{\pi}{4}, \epsilon^{2}\right) \\
(\pi, 0,0, \pi)\end{array}$ & $\begin{array}{l}\left(\frac{\pi}{4}, 0,0\right) \\
(0,0, \pi)\end{array}$ & $\begin{array}{l}\left(\frac{\pi}{4}, \epsilon^{2}, \epsilon^{2}\right) \\
(0,0,0,0)\end{array}$ \\
\hline 17 & $\begin{array}{l}\left(\epsilon^{2}, \epsilon, 1\right) \\
\left(\epsilon^{2}, \epsilon, 1\right)\end{array}$ & $\begin{array}{c}\left(\epsilon^{2}, \epsilon^{2}, \frac{\pi}{4}\right) \\
(\pi, \pi, \pi) \\
\end{array}$ & $\begin{array}{c}\left(\epsilon, 0, \epsilon^{2}\right) \\
(0,0,0) \\
\end{array}$ & $\begin{array}{c}\left(\frac{\pi}{4}, 0, \epsilon^{2}\right) \\
(0,0,0, \pi)\end{array}$ & $\begin{array}{c}\left(\epsilon^{2}, 0, \epsilon^{2}\right) \\
(0,0,0) \\
\end{array}$ & $\begin{array}{c}\left(\epsilon, 0, \epsilon^{2}\right) \\
(0,0,0, \pi)\end{array}$ \\
\hline 18 & $\begin{array}{l}\left(\epsilon^{2}, \epsilon, 1\right) \\
\left(\epsilon^{2}, \epsilon, 1\right)\end{array}$ & $\begin{array}{c}\left(\epsilon^{2}, \epsilon^{2}, \epsilon^{2}\right) \\
(\pi, 0, \pi)\end{array}$ & $\begin{array}{c}\left(\epsilon, 0, \epsilon^{2}\right) \\
(0,0,0)\end{array}$ & $\begin{array}{c}\left(\frac{\pi}{4}, \epsilon^{2}, \frac{\pi}{4}\right) \\
(\pi, 0, \pi, 0)\end{array}$ & $\begin{array}{c}\left(\epsilon, \epsilon^{2}, \epsilon^{2}\right) \\
(\pi, 0, \pi)\end{array}$ & $\begin{array}{c}\left(0, \epsilon^{2}, 0\right) \\
(0,0,0,0)\end{array}$ \\
\hline
\end{tabular}




\begin{tabular}{c|c|c|c|c|c|c}
\hline \multirow{2}{*}{19} & $(\epsilon, 1, \epsilon)$ & $\left(\epsilon^{2}, \epsilon^{2}, \epsilon^{2}\right)$ & $\left(\frac{\pi}{4}, 0, \epsilon^{2}\right)$ & $\left(\epsilon^{2}, \frac{\pi}{4}, \frac{\pi}{4}\right)$ & $(0, \epsilon, \epsilon)$ & $\left(\epsilon^{2}, \epsilon^{2}, \frac{\pi}{4}\right)$ \\
& $(\epsilon, 1,1)$ & $(\pi, \pi, \pi)$ & $(0,0,0)$ & $(\pi, 0,0,0)$ & $(0,0, \pi)$ & $(0,0,0,0)$ \\
\hline \multirow{2}{*}{20} & $\left(\epsilon^{2}, \epsilon, 1\right)$ & $\left(\epsilon^{2}, \epsilon^{2}, \epsilon^{2}\right)$ & $\left(\frac{\pi}{4}, 0,0\right)$ & $\left(\frac{\pi}{4}, \epsilon^{2}, \frac{\pi}{4}\right)$ & $\left(\epsilon^{2}, \epsilon^{2}, \epsilon^{2}\right)$ & $\left(\epsilon, \epsilon^{2}, \epsilon^{2}\right)$ \\
& $\left(\epsilon^{2}, \epsilon, 1\right)$ & $(0, \pi, \pi)$ & $(0,0,0)$ & $(\pi, 0,0, \pi)$ & $(0,0,0)$ & $(\pi, 0,0, \pi)$ \\
\hline \multirow{2}{*}{21} & $(\epsilon, 1, \epsilon)$ & $\left(\epsilon^{2}, \epsilon^{2}, \epsilon^{2}\right)$ & $\left(\frac{\pi}{4}, 0,0\right)$ & $\left(0, \epsilon^{2}, \frac{\pi}{4}\right)$ & $\left(\epsilon, \frac{\pi}{4}, \epsilon\right)$ & $\left(\epsilon^{2}, \epsilon, \epsilon^{2}\right)$ \\
& $(\epsilon, 1,1)$ & $(\pi, \pi, \pi)$ & $(0,0,0)$ & $(0,0,0,0)$ & $(0,0,0)$ & $(\pi, 0, \pi, 0)$ \\
\hline \multirow{2}{*}{22} & $\left(\epsilon^{2}, \epsilon, 1\right)$ & $\left(\epsilon^{2}, \epsilon^{2}, 0\right)$ & $\left(\frac{\pi}{4}, 0, \epsilon\right)$ & $\left(\frac{\pi}{4}, \epsilon^{2}, \frac{\pi}{4}\right)$ & $\left(\epsilon^{2}, \frac{\pi}{4}, \epsilon\right)$ & $\left(\epsilon^{2}, \frac{\pi}{4}, 0\right)$ \\
& $\left(\epsilon^{2}, \epsilon, 1\right)$ & $(0,0,0)$ & $(0,0,0)$ & $(\pi, 0,0,0)$ & $(0, \pi, 0)$ & $(0,0, \pi, 0)$ \\
\hline
\end{tabular}

Table 2: Supplementary information for the reconstruction of the mass matrices and Yukawa couplings of the models in Table 1. Note that we have made use of the freedom to set $\varphi_{i}^{\ell}=\varphi_{i}^{\ell^{\prime}}=\varphi_{i}^{D^{\prime}}=\alpha_{j}^{D}=0$ for $i=1,2,3$ and $j=1,2$.

\section{Acknowledgments}

The research of F.P. is supported by Research Training Group 1147 Theoretical Astrophysics and Particle Physics of Deutsche Forschungsgemeinschaft. G.S. is supported by the Federal Ministry of Education and Research (BMBF) under contract number 05HT1WWA2. W.W. would like to acknowledge support from the Emmy Noether program of Deutsche Forschungsgemeinschaft.

\section{References}

[1] S. Fukuda et al. [Super-Kamiokande Collaboration], Phys. Lett. B 539, 179 (2002) [arXiv:hep-ex/0205075]; Q. R. Ahmad et al. [SNO Collaboration], Phys. Rev. Lett. 89, 011302 (2002) [arXiv:nucl-ex/0204009].

[2] Y. Fukuda et al. [Super-Kamiokande Collaboration], Phys. Rev. Lett. 81, 1562 (1998) [arXiv:hep-ex/9807003].

[3] T. Araki et al. [KamLAND Collaboration], Phys. Rev. Lett. 94, 081801 (2005) [arXiv:hep-ex/0406035]; M. Apollonio et al. [CHOOZ Collaboration], Eur. Phys. J. C 27, 331 (2003) [arXiv:hep-ex/0301017].

[4] E. Aliu et al. [K2K Collaboration], Phys. Rev. Lett. 94, 081802 (2005) [arXiv:hepex/0411038].

[5] H. Georgi and S. L. Glashow, Phys. Rev. Lett. 32, 438 (1974); H. Georgi, in Proceedings of Coral Gables 1975, Theories and Experiments in High Energy Physics, New York, 1975.

[6] J. C. Pati and A. Salam, Phys. Rev. D 10, 275 (1974) [Erratum-ibid. D 11, 703 (1975)]. 
[7] P. Minkowski, Phys. Lett. B 67, 421 (1977); T. Yanagida, in Proceedings of the Workshop on the Unified Theory and Baryon Number in the Universe, KEK, Tsukuba, 1979; M. Gell-Mann, P. Ramond and R. Slansky, in Proceedings of the Workshop on Supergravity, Stony Brook, New York, 1979; S. L. Glashow, in Proceedings of the 1979 Cargese Summer Institute on Quarks and Leptons, New York, 1980.

[8] M. Magg and C. Wetterich, Phys. Lett. B 94, 61 (1980); R. N. Mohapatra and G. Senjanović, Phys. Rev. Lett. 44, 912 (1980); Phys. Rev. D 23, 165 (1981); J. Schechter and J. W. F. Valle, Phys. Rev. D 22, 2227 (1980); G. Lazarides, Q. Shafi and C. Wetterich, Nucl. Phys. B 181, 287 (1981).

[9] H. Georgi and H. Quinn, Phys. Rev. Lett. 33, 451 (1974); S. Dimopoulos, S. Raby and F. Wilczek, Phys. Rev. D 24, 1681 (1981); S. Dimopoulos and H. Georgi, Nucl. Phys. B 193, 150 (1981).

[10] T. Schwetz, Phys. Scripta T127, 1 (2006) [arXiv:hep-ph/0606060].

[11] B. Pontecorvo, Sov. Phys. JETP 6, 429 (1957); Z. Maki, M. Nakagawa and S. Sakata, Prog. Theor. Phys. 28, 870 (1962).

[12] P. F. Harrison, D. H. Perkins and W. G. Scott, Phys. Lett. B 458, 79 (1999) [arXiv:hepph/9904297]; P. F. Harrison, D. H. Perkins and W. G. Scott, Phys. Lett. B 530, 167 (2002) [arXiv:hep-ph/0202074].

[13] F. Plentinger and W. Rodejohann, Phys. Lett. B 625, 264 (2005) [arXiv:hep$\mathrm{ph} / 0507143]$.

[14] D. Majumdar and A. Ghosal, Phys. Rev. D 75, 113004 (2007) [arXiv:hep-ph/0608334]; A. H. Chan, H. Fritzsch, S. Luo and Z. z. Xing, Phys. Rev. D 76, 073009 (2007) [arXiv:0704.3153 [hep-ph]]; S. F. King, Phys. Lett. B 659, 244 (2008) [arXiv:0710.0530 [hep-ph]].

[15] Z. z. Xing, Phys. Lett. B 533, 85 (2002) [arXiv:hep-ph/0204049]; Z. z. Xing, H. Zhang and S. Zhou, Phys. Lett. B 641, 189 (2006) [arXiv:hep-ph/0607091]; E. Ma, arXiv:0709.0507 [hep-ph]; A. Mondragon, M. Mondragon and E. Peinado, arXiv:0712.2488 [hep-ph].

[16] G. Altarelli, arXiv:0705.0860 [hep-ph].

[17] N. Cabibbo, Phys. Rev. Lett. 10, 531 (1963); M. Kobayashi and T. Maskawa, Prog. Theor. Phys. 49, 652 (1973).

[18] E. Ma, Mod. Phys. Lett. A 17, 627 (2002) [arXiv:hep-ph/0203238]; G. Altarelli and F. Feruglio, Nucl. Phys. B 741, 215 (2006) [arXiv:hep-ph/0512103]; S. F. King and M. Malinsky, JHEP 11, 071 (2006) [arXiv:hep-ph/0608021]; Phys. Lett. B 645, 351 (2007) [arXiv:hep-ph/0610250]; F. Feruglio, C. Hagedorn, Y. Lin and L. Merlo, Nucl. Phys. B 775, 120 (2007) [arXiv:hep-ph/0702194]; C. Luhn, S. Nasri and P. Ramond, Phys. Lett. B 652, 27 (2007) [[arXiv:0706.2341 [hep-ph]]. 
[19] E. Ma, H. Sawanaka and M. Tanimoto, Phys. Lett. B 641, 301 (2006) [arXiv:hepph/0606103]; I. de Medeiros Varzielas, S. F. King and G. G. Ross, Phys. Lett. B 648, 201 (2007) [arXiv:hep-ph/0607045]; E. Ma, Mod. Phys. Lett. A 21, 2931 (2006) [arXiv:hepph/0607190]; S. Morisi, M. Picariello and E. Torrente-Lujan, Phys. Rev. D 75, 075015 (2007) [arXiv:hep-ph/0702034]; M.-C. Chen and K. T. Mahanthappa, [arXiv:0705.0714 [hep-ph]]; G. Altarelli, F. Feruglio and C. Hagedorn, arXiv:0802.0090 [hep-ph].

[20] A. Y. Smirnov, arXiv:hep-ph/0402264; M. Raidal, Phys. Rev. Lett. 93, 161801 (2004) [arXiv:hep-ph/0404046]; H. Minakata and A. Y. Smirnov, Phys. Rev. D 70, 073009 (2004) [arXiv:hep-ph/0405088].

[21] M. Jezabek and Y. Sumino, Phys. Lett. B 457, 139 (1999) [arXiv: hep-ph/9904382]; C. Giunti and M. Tanimoto, Phys. Rev. D 66, 113006 (2002) [arXiv:hep-ph/0209169]; P. H. Frampton, S. T. Petcov and W. Rodejohann, Nucl. Phys. B 687, 31 (2004) [arXiv:hep-ph/0401206].

[22] T. Ohlsson, Phys. Lett. B 622, 159 (2005) [arXiv:hep-ph/0506094]; S. Antusch and S. F. King, Phys. Lett. B 631, 42 (2005) [arXiv:hep-ph/0508044].

[23] K. Cheung, S. K. Kang, C. S. Kim and J. Lee, Phys. Rev. D 72, 036003 (2005) [arXiv:hep-ph/0503122]; K. A. Hochmuth and W. Rodejohann, Phys. Rev. D 75, 073001 (2007) [arXiv:hep-ph/0607103].

[24] W. Rodejohann, Phys. Rev. D 69, 033005 (2004) [arXiv:hep-ph/0309249]; N. Li and B.Q. Ma, Phys. Rev. D 71, 097301 (2005) [arXiv:hep-ph/0501226]; Z.-z. Xing, Phys. Lett. B 618, 141 (2005) [arXiv:hep-ph/0503200]; A. Datta, L. L. Everett and P. Ramond, Phys. Lett. B 620, 42 (2005) [arXiv:hep-ph/0503222]; L. L. Everett, Phys. Rev. D 73, 013011 (2006) [arXiv:hep-ph/0510256].

[25] B. C. Chauhan, M. Picariello, J. Pulido and E. Torrente-Lujan, Eur. Phys. J. C 50, 573 (2007) [arXiv:hep-ph/0605032].

[26] A. Dighe, S. Goswami and P. Roy, Phys. Rev. D 73, 071301 (2006) [arXiv:hepph/0602062]; M. A. Schmidt and A. Y. Smirnov [arXiv:hep-ph/0607232].

[27] T. Ohlsson and G. Seidl, Nucl. Phys. B 643, 247 (2002) [arXiv:hep-ph/0206087]; P. H. Frampton and R. N. Mohapatra, JHEP 01, 025 (2005) [arXiv:hep-ph/0407139]; S. Antusch, S. F. King and R. N. Mohapatra, Phys. Lett. B 618, 150 (2005) [arXiv:hepph/0504007]; M. Picariello, hep-ph/0611189; A. Hernandez-Galeana, Phys. Rev. D 76, 093006 (2007) [arXiv:0710.2834 [hep-ph]].

[28] F. Plentinger, G. Seidl and W. Winter, Nucl. Phys. B 791, 60 (2008) [arXiv:hep$\mathrm{ph} / 0612169]$.

[29] F. Plentinger, G. Seidl and W. Winter, Phys. Rev. D 76, 113003 (2007) [arXiv:0707.2379 [hep-ph]].

[30] W. Winter, Phys. Lett. B 659, 275 (2008) [arXiv:0709.2163 [hep-ph]]. 
[31] G. C. Branco, D. Emmanuel-Costa, R. Gonzalez Felipe and H. Serodio, arXiv:0711.1613 [hep-ph]; G. C. Branco, D. Emmanuel-Costa, M. N. Rebelo and P. Roy, arXiv:0712.0774 [hep-ph].

[32] C. D. Froggatt and H. B. Nielsen, Nucl. Phys. B 147, 277 (1979).

[33] L. M. Krauss and F. Wilczek, Phy. Rev. Lett. 62, 1221 (1989).

[34] J. Sayre and S. Wiesenfeldt, arXiv:0711.1687 [hep-ph].

[35] F. Feruglio and Y. Lin, arXiv:0712.1528 [hep-ph].

[36] S. Antusch, P. Huber, J. Kersten, T. Schwetz and W. Winter, Phys. Rev. D 70, 097302 (2004) [arXiv:hep-ph/0404268].

[37] R. Gandhi and W. Winter, Phys. Rev. D 75, 053002 (2007) [arXiv:hep-ph/0612158].

[38] F. Plentinger and G. Seidl, in preparation. 